\title{
Mechanical properties of silicone composites reinforced with micron- and nano-sized magnetic particles
}

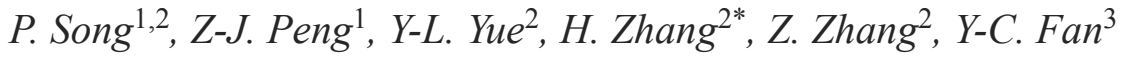 \\ ${ }^{1}$ School of Engineering and Technology, China University of Geosciences at Beijing, 100083 Beijing, China \\ ${ }^{2}$ National Center for Nanoscience and Technology, 100190 Beijing, China \\ ${ }^{3}$ University of Science and Technology of China, 230027 Hefei, China
}

Received 26 December 2012; accepted in revised form 5 March 2013

\begin{abstract}
Silicone composites filled with different-sized nickel particles were prepared. By applying a permanent magnet, both the micron- and nano-sized particles were found to distribute along the magnetic field direction, resulting in chain-like microstructures, which improved the key mechanical properties of the resultant samples effectively, compared to the samples with randomly-distributed particles. The composites were also tested under various magnetic field strengths. The samples with aligned particles showed larger improvements in shear storage modulus than those with random particles.
\end{abstract}

Keywords: polymer composites, nickel particles, mechanical properties, alignment

\section{Introduction}

Owing to their unique properties such as electrical insulating property, high weather resistance, good biocompatibility as well as physiological inertness, silicone rubbers gain numerous applications in practice. However, as compared to other rubbers (e.g. natural rubber, styrene butadiene rubber), silicone rubbers have relatively weak mechanical properties, so that their applications are limited significantly; this case is especially serious for the room temperature vulcanized silicone rubbers (RTV), since they possess low crosslink density in comparison with the high temperature vulcanized silicones (HTV). To reinforce RTV, the common way is to introduce nano-sized rigid inorganic particles to it, which include $\mathrm{SiO}_{2}, \mathrm{Al}_{2} \mathrm{O}_{3}, \mathrm{CaCO}_{3}, \mathrm{TiO}_{2}$, carbon black etc. [1-5]. Normally, reinforcing particles are randomly distributed in RTV without any alignment. If the particles can be aligned to form fiberlike structure, they would result in more significant reinforcing effects, as well as enhancements in elec- trical and thermal conductivity along the aligning direction, provided that the particles are electrically or thermally conductive in nature [6-13]. Actually, force, electrical and magnetic fields are normally applied to make particles align in polymers. For example, at the temperatures above their melt points thermoplastics can be drawn to polymer fibers or films with applied force; meantime, the particles in the polymers (e.g. carbon nanotubes, layered fillers) are able to align along the force direction. It has been reported that the force field could be also applied to thermosetting polymers for aligning particles, when the thermosetting polymers are semicured, showing the ability to undergo large deformation $[14,15]$. Note this method is only used for manufacturing polymer fibers or films. Instead, external electrical or magnetic fields can be applied to make particle alignment in bulk polymers [16-24].

In this work we reinforced RTV composite samples filled with micro- or nano- nickel (Ni) particles. By applying a magnetic field, both micro- and nanopar-

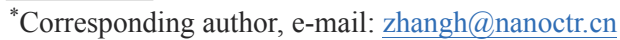

(C) BME-PT
} 
ticles can align along the direction of the magnetic field. The key mechanical properties of the siliconebased composites were compared with/without applying the magnetic field.

\section{Experimental}

Hydroxyl-terminated ploydimethysiloxane prepolymer (molecular weight $=20000$, Wacker Chemie AG, Germany) was used as polymer matrix in this study. Both micro- and nano- Ni particles were provided by Hebao Nanomaterial Co. Ltd, China. Their SEM images are shown in Figure 1, respectively. According to the SEM images, the diameters of the micro-particles and nanoparticles were measured to be in the range of 2-10 $\mu \mathrm{m}$ and $100-200 \mathrm{~nm}$, respectively.

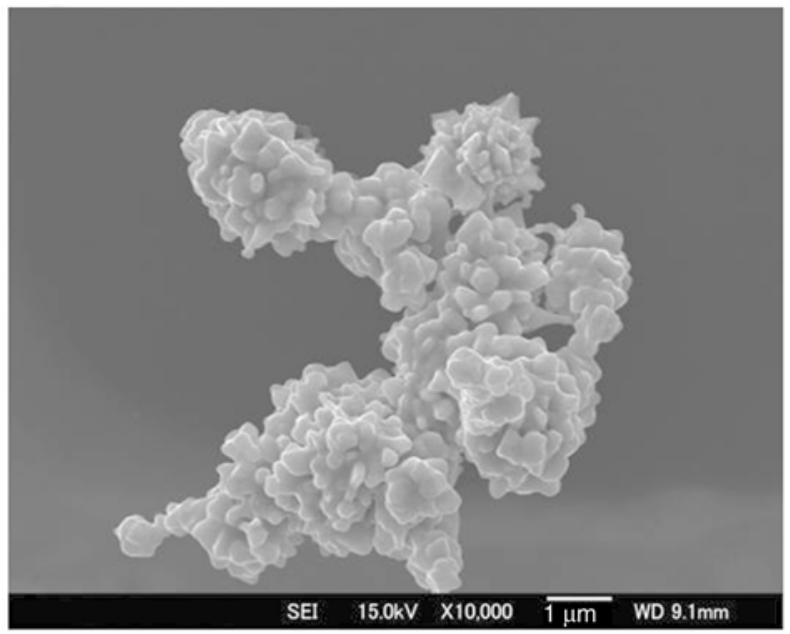

a)
The Ni particles were mixed with the ploydimethysiloxane prepolymer and stirred intensively by a high-speed dissolver (DISPERMAT AE, Germany) at a speed of $5000 \mathrm{rpm}$ for $1 \mathrm{~h}$. The mixture was further processed by a three-roll mill machine (EXAKT $80 \mathrm{E}$, Germany) at room temperature for $30 \mathrm{~min}$, followed by adding appropriate amounts of curing agent (TEOS) and accelerator (dibutyltin dilaurate); accordingly, the mixture was poured into a rectangular PTFE mold $(120 \mathrm{~mm} \times 120 \mathrm{~mm} \times 3 \mathrm{~mm})$, which was placed between a pair of permanent magnets with the magnetic field of $0.05 \mathrm{~T}$. Figure 2 shows the experimental setup and related photo. In some cases, xylene was added to the mixture so as to reduce viscosity of the mixture and promote mobility of Ni particles under magnetic field. The mix-

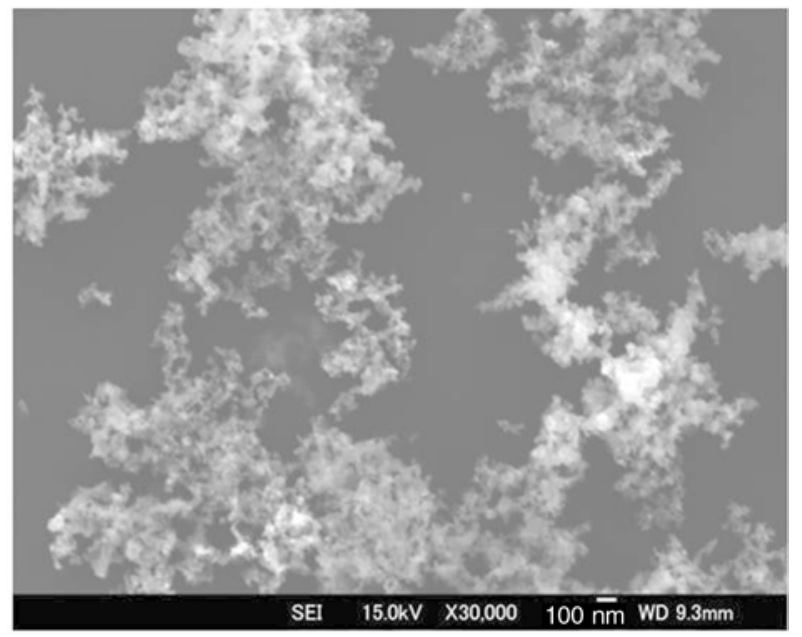

b)

Figure 1. SEM micrographs of Ni particles: (a) micro-particles, (b) nanoparticles

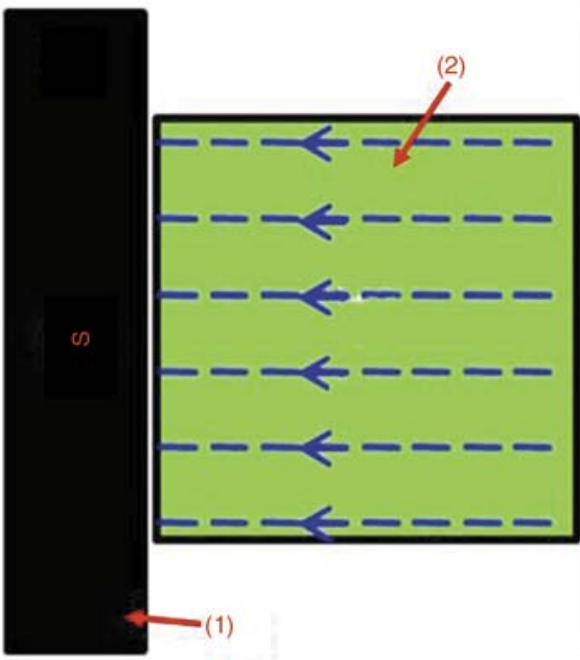

a)

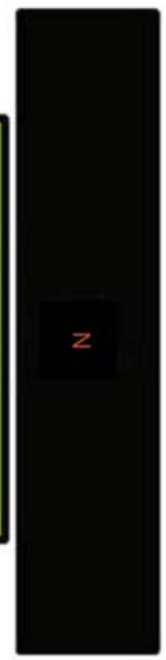

b)

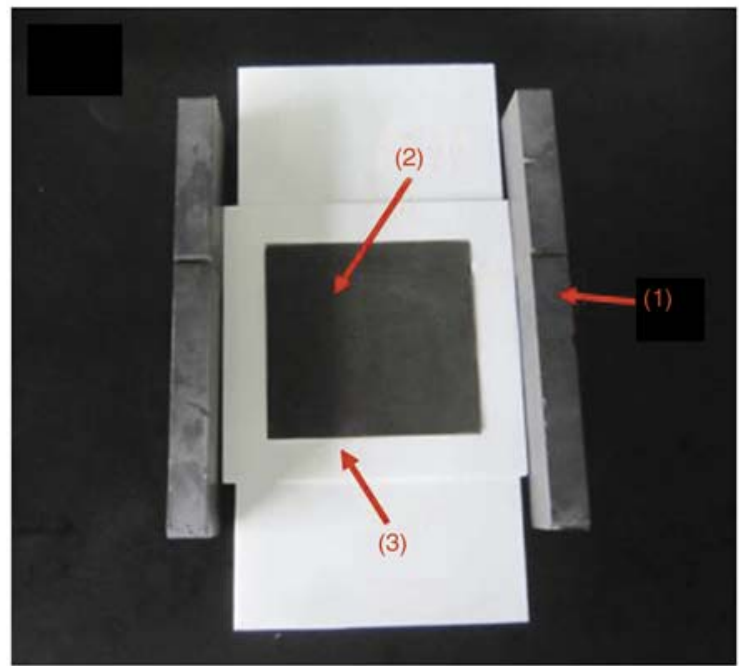

(1) permanent magnet

(2) uncured mixture
(3) PTFE mold

Figure 2. Configuration of preparing silicone composite samples with aligned particles: (a) a schematic diagram and (b) a photo 
ture was cured at room temperature for several days until complete cure.

The alignment process of Ni particles in the prepolymer was in-situ observed by a transmission optical microscope (TOM, Leica DM4000M, Germany). Additionally, the cured samples were placed into liquid nitrogen and then fractured along the alignment direction; the broken surfaces were observed by a scanning electron microscope (SEM, Hitachi S-4800, Japan).

The mechanical properties of composite samples were measured with/without applying magnetic field. Tensile test and tear test of the composite samples were performed without applying magnetic field, using a SANS CMT2000 Tester (Shenzhen Sans Material Test Instrument Co., Ltd., China) at a crosshead speed of $50 \mathrm{~mm} / \mathrm{min}$ in accordance to GB/T-528. Dynamic mechanical properties of these samples were measured by a dynamic mechanical analyzer (Triton Technology Ltd, UK, model Tritec 2000B) coupled with a self-made electromagnet that can generate a variable magnetic field from 0 to $1000 \mathrm{mT}$. The sample had dimensions of $10 \mathrm{~mm} \times$
$3 \mathrm{~mm} \times 3 \mathrm{~mm}$, in which the particle chains (if they were aligned) were distributed along the thickness direction. The DMA test was performed at room temperature, in shear mode with the shear strain amplitude of $0.1 \%$. The detail of experiment configuration is schematically shown in Figure 3.

\section{Results and discussion}

\subsection{Alignment process of micro- and} nano-sized $\mathrm{Ni}$ particles in silicone resin

The viscosity of the Ni/RTV mixture has significant influence on the alignment process of Ni particles. Takahashi et al. [25] found in the vapor-grown carbon fiber/silicone oil systems the fiber alignment and the subsequent network formation was almost proportional to the matrix viscosity, because the high viscosity can effectively impede the fiber rotation and movement under dc electric field. In the present work, the rheological behavior of the Ni/RTV mixture was measured and shown in Figure 4. As seen, the micro-particles only slightly increase the viscosity of the mixture, compared to the nanoparticles at the same filler content. This is because that the

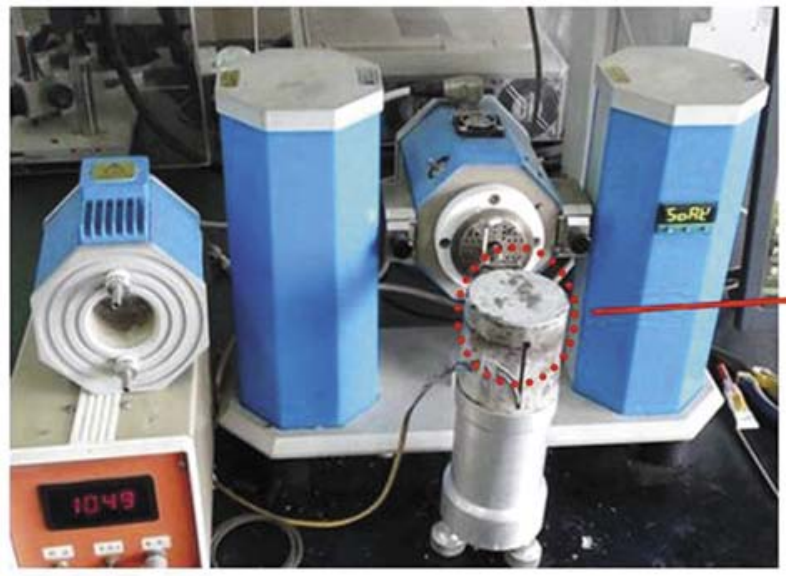

a)

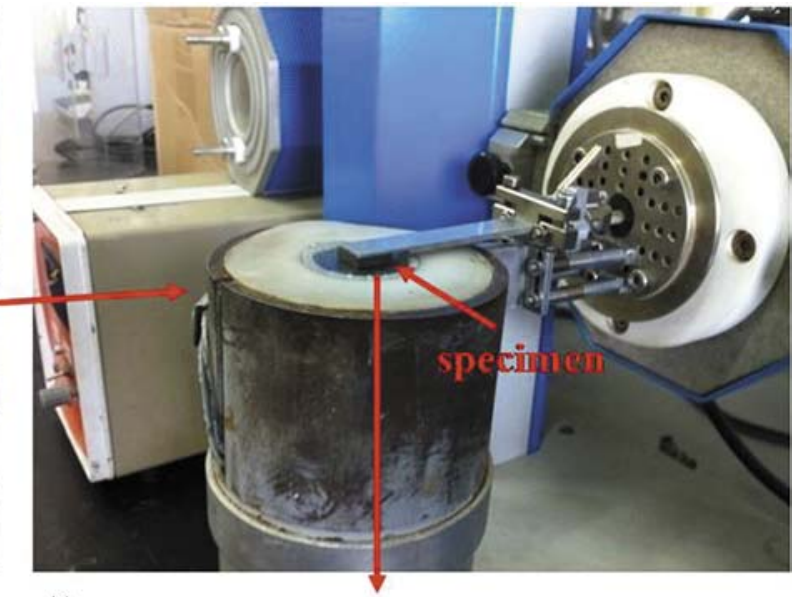

b)
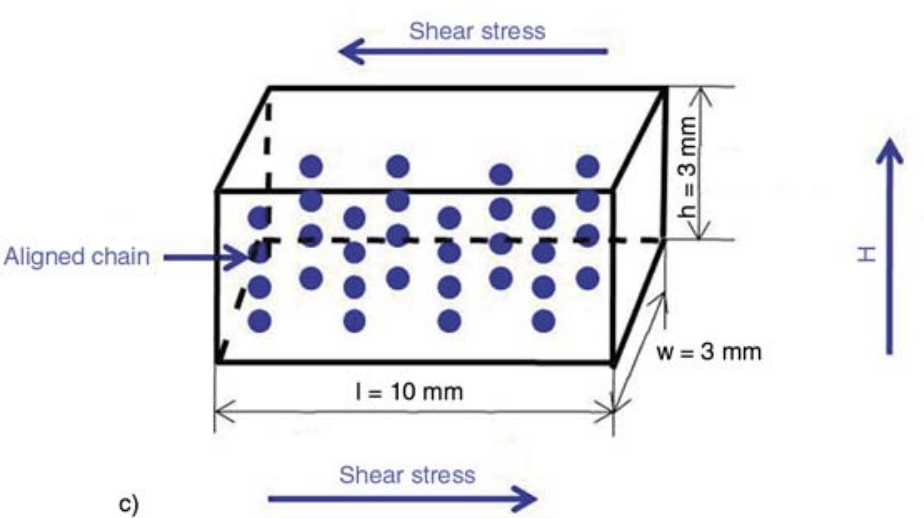

Figure 3. Configuration of measuring the shear storage modulus of silicone composite samples: (a) a photo of the modified DMA, (b) a photo of specimen and (c) a schematic diagram of specimen 


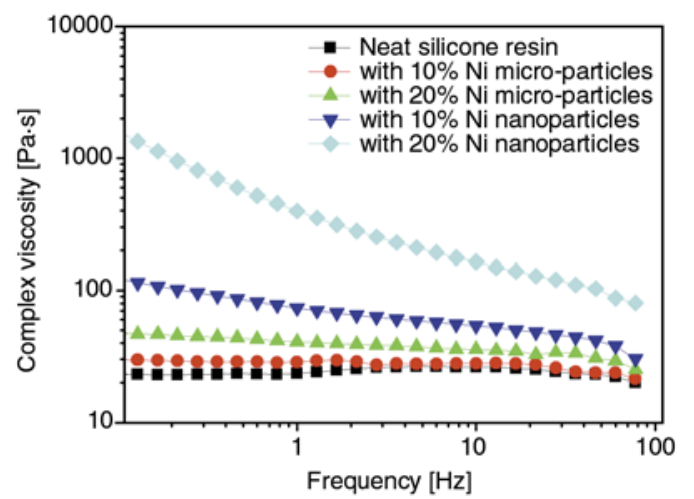

Figure 4. Complex viscosities of neat RTV resin and RTV composites with micro- or nanoparticles

nanoparticles have much larger specific surface area, which may interact with the matrix during flow. At high filler content of $20 \mathrm{wt} \%$, the nanoparticle/RTV mixture is found to present obvious thixotropic effect.

The mixture of micron-sized $\mathrm{Ni}$ /uncured resin is placed under a magnetic field and in-situ observed by TOM to study the alignment process of particles.

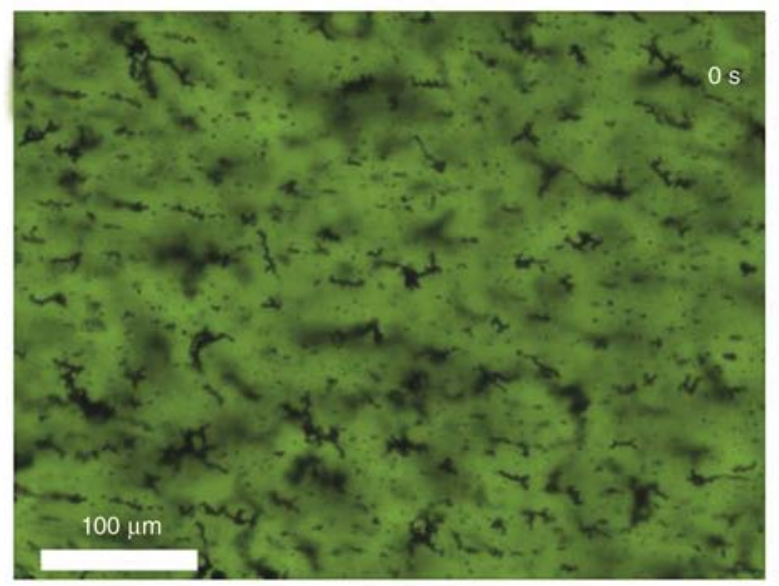

a)

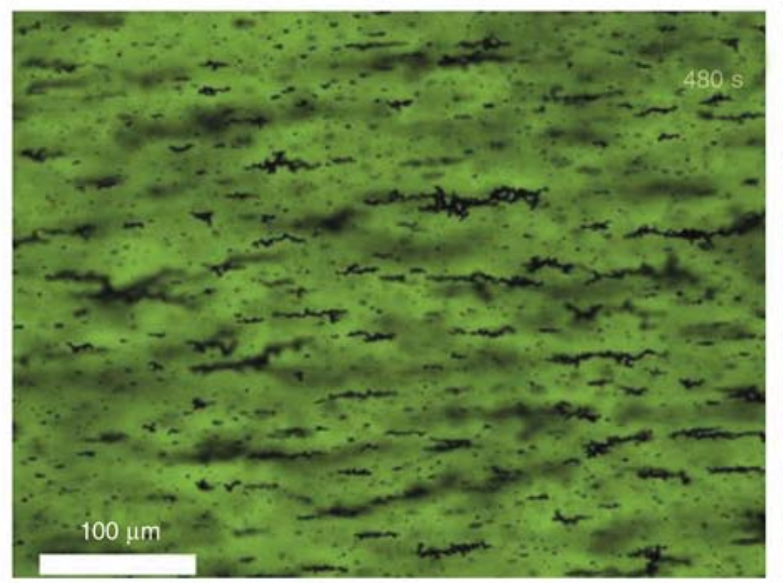

c)
At a filler content of $5 \mathrm{wt} \%$ (Figure $5 \mathrm{a}-5 \mathrm{~d}$ ), the chainlike microstructures begin to form at the time of $120 \mathrm{~s}$ (Figure 5b); with further increasing the time up to $480 \mathrm{~s}$, the chain-like microstructures become longer and clearer (Figure 5c); after $600 \mathrm{~s}$, the amount and dimensions of the chain-like microstructures are no longer changed (Figure 5d), suggesting the alignment process finishes. Figure 6 presents the relationship between the time to finish alignment process, $T_{\mathrm{f}}$, and the filler content. It can be seen that with increasing of filler content, the $T_{\mathrm{f}}$ value increases significantly. This phenomenon is easy to understand. At higher filler content, the blend becomes more viscous (Figure 4); the high viscosity will prevent the Ni particles from alignment and therefore, increase the time to finish alignment.

Regarding the nano-sized Ni particles, it is difficult to observe their distribution with TOM due to their small size and the low optical transparency of the uncured nanocomposite sample. However, their distribution can be observed by SEM after the mixture

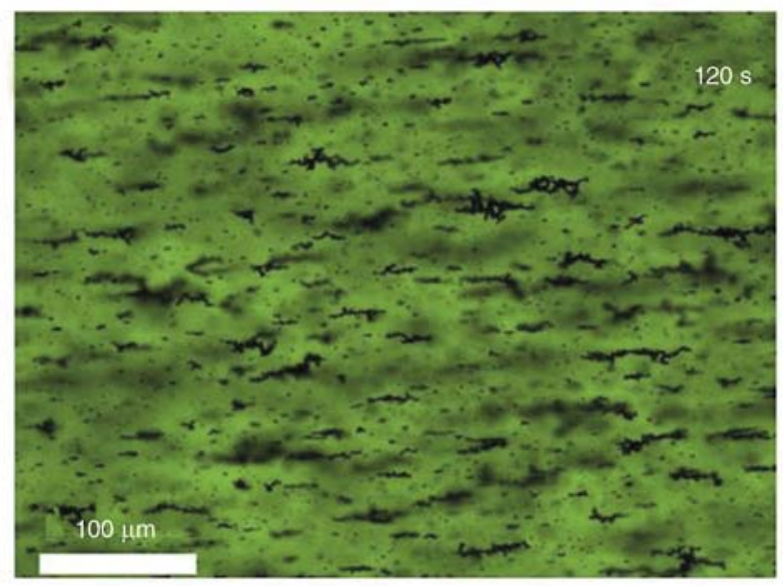

b)

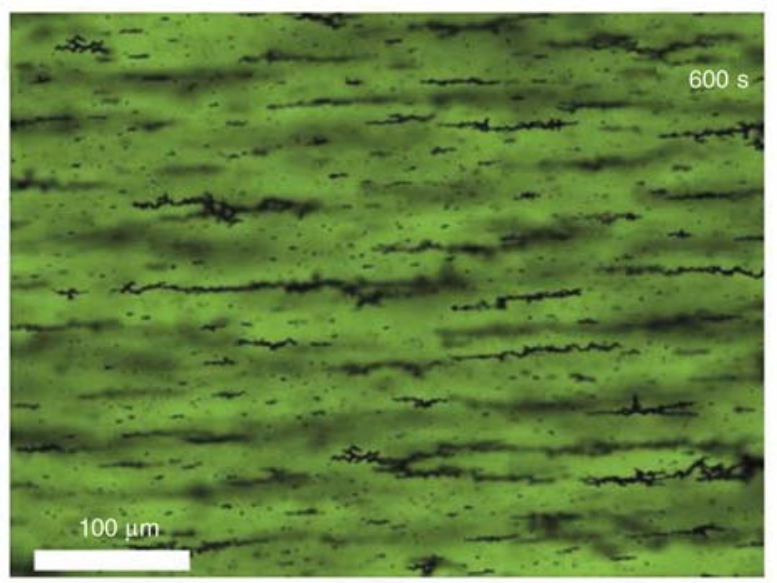

d)

Figure 5. Optical microscope images showing the alignment of Ni micro-particles ( $5 \mathrm{wt} \%)$ in RTV resin at various times: (a) $0 \mathrm{~s}$, (b) $120 \mathrm{~s}$, (c) $480 \mathrm{~s}$ and (d) $600 \mathrm{~s}$ 


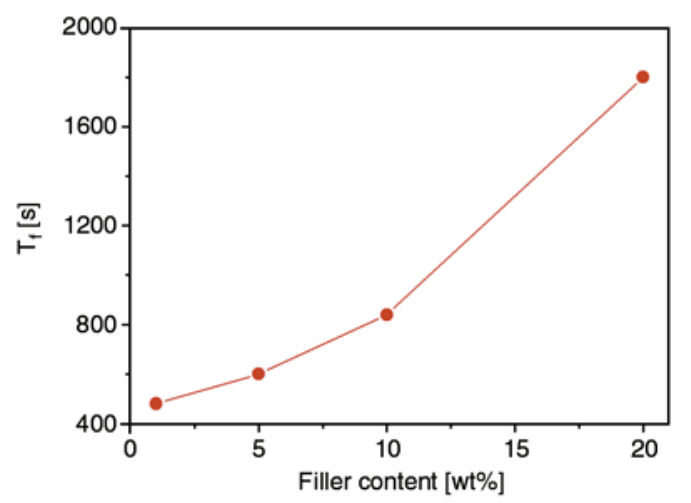

Figure 6. The relationship between time to finish alignment process and filler content

is fully cured. Figure 7a shows the distribution of the nanoparticles in the absence of the magnetic field. It can be seen that the nano-sized Ni particles randomly distribute in the silicone matrix; in addition, some agglomerates still exist in the RTV matrix. However, when the magnetic field is applied to the mixture, the nanoparticles appear to form the chainlike microstructure (Figure 7b), as indicated by the arrows.

\subsection{Mechanical properties of silicone composites}

\subsubsection{At zero magnetic field}

We measured the key mechanical properties of the RTV samples filled with micron- and nano-sized Ni particles without application of magnetic field. The results are shown in Figure 8. The modulus at 100\% strain, tensile strength, elongation at break as well as tear strength of the RTV samples increase rapidly with increasing of the filler content, regardless of the filler size and alignment. The nano-sized Ni particles exhibit much better reinforcing effects than

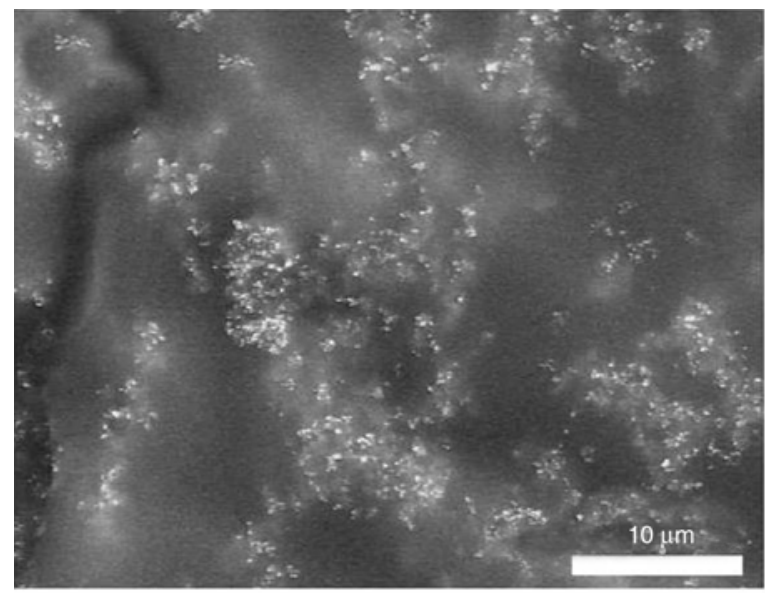

a) the micron-sized ones at different filler contents. This is due to the fact that the former has much larger specific surface area than the latter, and therefore the nanoparticles shows stronger interactions with RTV polymer. Moreover, the alignment of particles is found to improve the mechanical properties of the RTV samples further, even though the effect is relatively minor. As an example of the RTV samples containing $30 \mathrm{wt} \%$ nanoparticles, the improvements in modulus, tensile strength, elongation at break and tear strength are 3.7, 10.3, 7.2 and $5.0 \%$, respectively, for the aligned samples than for the random samples.

\subsubsection{At various magnetic fields}

We also studied the mechanical properties of RTV composite samples tested at various magnetic field strengths. Due to the limitation of research conditions, only the shear storage modulus was measured; the test configuration is illustrated in Figure 3. For the purpose of comparison, the shear storage modulus of samples measured at zero field (without magnetic field) is also plotted in Figure 9a. As seen in this figure, the shear storage moduli of samples develop a tendency similar to that of the tensile modulus and tensile strength (Figure 8), that means: (i) the shear storage modulus increases with the filler content; (ii) the nanocomposites show larger shear storage modulus than the micro-composites at given filler content; (iii) the samples with aligned particles show higher shear storage modulus than those with random particles. When a magnetic field $(1000 \mathrm{mT})$ is applied to the samples, the basic tendency as mentioned above does not appear to change significantly, as shown in Figure 9b; however, if

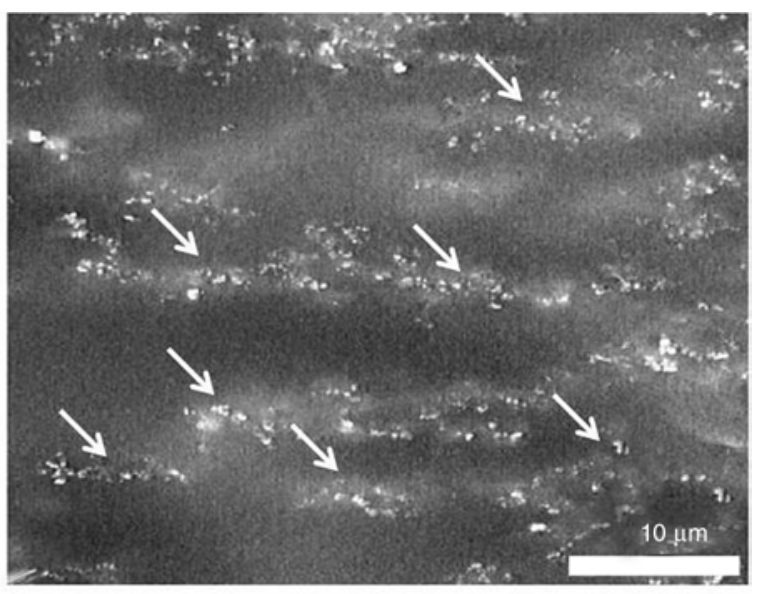

b)

Figure 7. SEM micrographs of Ni nanoparticles in RTV composites (a) without magnetic field and (b) with magnetic field 

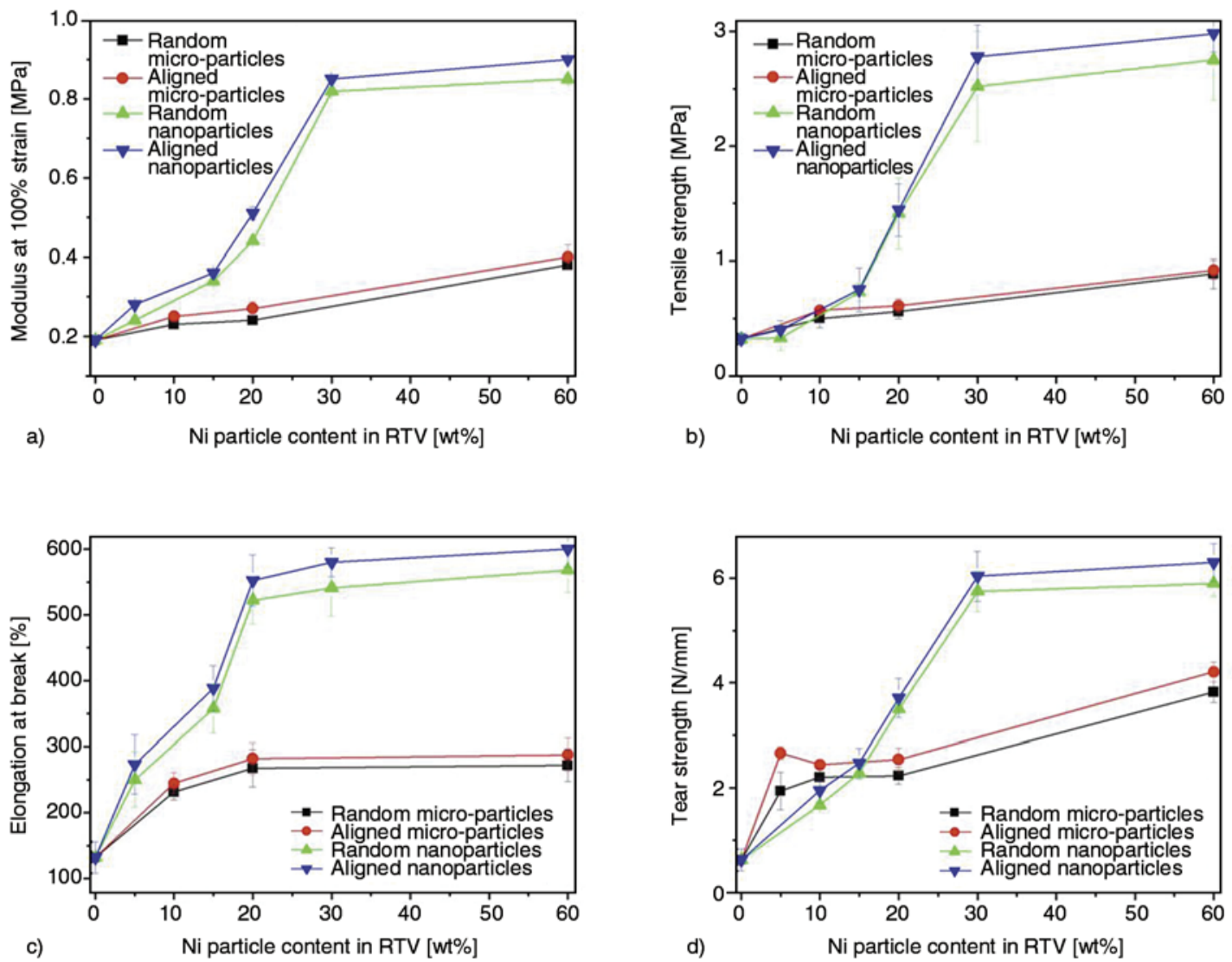

Figure 8. Key mechanical properties of RTV composite samples measured in the absence of magnetic field (a) modulus at $100 \%$ strain, (b) tensile strength, (c) elongation at break and (d) tear strength
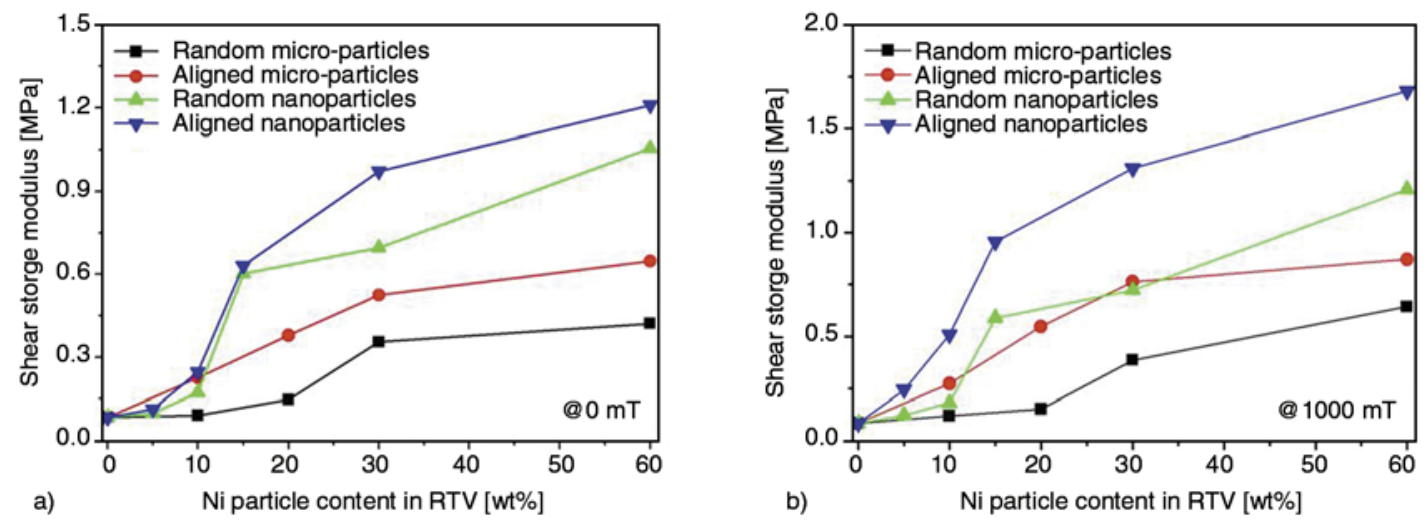

Figure 9. Comparison of shear storage modulus of RTV samples measured under (a) zero and (b) 1000 mT magnetic fields. The test frequency was $10 \mathrm{~Hz}$

comparing the Figure 9a with Figure $9 \mathrm{~b}$ carefully, one can find that application of the magnetic field can further enhance the shear storage modulus. As an example of the RTV samples containing $30 \mathrm{wt} \%$ randomly-distributed nanoparticles, its shear storage modulus is $0.69 \mathrm{MPa}$ at zero field, however, this value becomes $0.73 \mathrm{MPa}$ at the magnetic field of $1000 \mathrm{mT}$, i.e. slight improvement is achieved.

In order to better study the effect of magnetic field strength on the shear modulus of samples studied, their relationship is plotted in Figure 10. Here, only the results of the RTV samples containing $30 \mathrm{wt} \%$ micron- or nanoparticles are plotted. It is interesting to find from this figure that with increasing the magnetic field strength, the shear storage modulus improves more significantly for the samples containing the aligned particles than for the samples containing the random particles. As the magnetic field strength ranges from 0 to $1000 \mathrm{mT}$, the shear storage modulus of the sample with aligned nanoparticles increases by $35 \%$ (from 0.97 to $1.31 \mathrm{MPa}$ ), however for the sample with random nanoparticles 


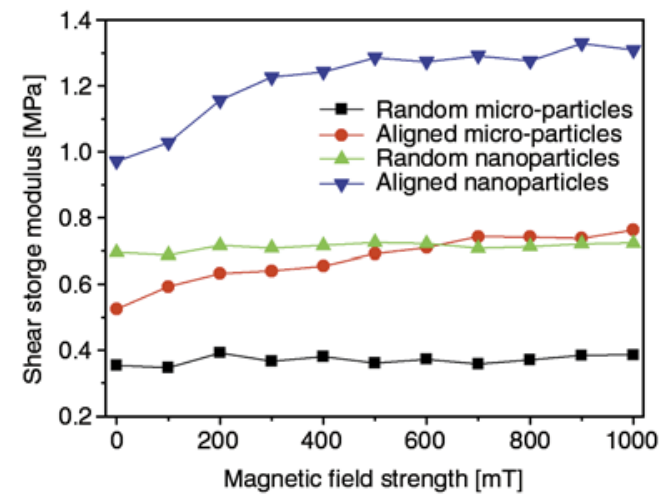

Figure 10. The effect of magnetic field strength on shear storage modulus of RTV composite samples

only 4\% increase is achieved (from 0.69 to $0.73 \mathrm{MPa}$ ). Similar phenomenon is also found for the samples containing micro-particles. Having the chain-like structure, the aligned particles could be united more strongly under magnetic fields and play the role like short fibers, therefore, the stress transfer from matrix to particles could be promoted efficiently. Comparatively, for the random particles, the stress transfer level would be lower due to their unordered structure.

\section{Conclusions}

RTV-based composite samples filled with differentsized Ni particles were prepared. Under magnetic field, both micron- and nano-sized particles aligned along the magnetic field direction and thus forming chain-like micro-structures, favoring the stress transfer from matrix to particles. The aligning process occurred easily at low filler content (i.e. lower viscosity). In comparison with the micro-composites, the nanocomposites had the better mechanical properties in the absence of magnetic fields, which including tensile modulus, tensile strength, elongation at break as well as tear strength. Moreover, the alignment of particles indeed improved these key mechanical properties further, even though the effects are relatively mild. In the presence of magnetic fields, the samples with the aligned particles showed faster improvements in shear storage modulus than those with the random particles.

\section{Acknowledgements}

This work was supported by the National High Technology Research and Development Program of China (863 program, No.2013AA031803). The authors thank Prof. Xinglong Gong (University of Science and Technology of China) for the assistance during the experimental work.

\section{References}

[1] Schaefer D. W., Vu B. T. N., Mark J. E.: The effect of interphase coupling on the structure and mechanical properties of silica-siloxane composites. Rubber Chemistry and Technology, 75, 795-810 (2002). DOI: $10.5254 / 1.3547684$

[2] Xu J., Wong C. P.: Low-loss percolative dielectric composite. Applied Physics Letters, 87, 082907/1082907/3 (2005).

DOI: $10.1063 / 1.2032597$

[3] Kaully T., Siegmann A., Shacham D.: Mechanical behavior of highly filled natural $\mathrm{CaCO}_{3}$ composites: Effect of particle size distribution and interface interactions. Polymer Composites, 29, 396-408 (2008). DOI: $10.1002 / p c .20435$

[4] Carpi F., Rossi D. D.: Improvement of electromechanical actuating performances of a silicone dielectric elastomer by dispersion of titanium dioxide powder. IEEE Transactions on Dielectrics and Electrical Insulation, 12, 835-843 (2005).

DOI: $10.1109 /$ TDEI.2005.1511110

[5] Dang Z-M., Xia B., Yao S-H., Jiang M-J., Song H-T., Zhang L-Q., Xie D.: High-dielectric-permittivity highelasticity three-component nanocomposites with low percolation threshold and low dielectric loss. Applied Physics Letters, 94, 042902/1-042902/3 (2009).

DOI: $10.1063 / 1.3072355$

[6] Cho H-B., Tokoi Y., Tanaka S., Suematsu H., Suzuki T., Jiang W., Niihara K., Nakayama T.: Modification of BN nanosheets and their thermal conducting properties in nanocomposite film with polysiloxane according to the orientation of BN. Composites Science and Technology, 71, 1046-1052 (2011). DOI: 10.1016/j.compscitech.2011.03.002

[7] Cho H-B., Nakayama T., Suzuki T., Tanaka S., Jiang W., Suematsu H., Niihara K.: Linear assembles of BN nanosheets, fabricated in polymer/BN nanosheet composite film. Journal of Nanomaterials, 2011, 7-13 (2011). DOI: $10.1155 / 2011 / 693454$

[8] Zhang B., Li B., Xie C.: Epoxy resin/nano Ni@C composites exhibiting NTC effect with tunable resistivity. Journal of Materials Science and Technology, 25, 159163 (2009).

[9] Mahfuz H., Zainuddin S., Parker M. R., Al-Saadi T., Rangari V. K., Jeelani S.: Reinforcement of SC-15 epoxy with CNT/CNF under high magnetic field: An investigation of mechanical and thermal response. Journal of Materials Science, 44, 1113-1120 (2009). DOI: $10.1007 / \mathrm{s} 10853-008-3161-5$

[10] Wang Q., Dai J., Li W., Wei Z., Jiang J.: The effects of CNT alignment on electrical conductivity and mechanical properties of SWNT/epoxy nanocomposites. Composites Science and Technology, 68, 16441648 (2008).

DOI: 10.1016/j.compscitech.2008.02.024 
[11] Leng J., Lan X., Liu Y. J., Du S., Huang W. M., Liu N., Phee S. J., Yuan Q.: Electrical conductivity of thermoresponsive shape-memory polymer with embedded micron sized Ni powder chains. Applied Physics Letters, 92, 014104/1-014104/3 (2008).

DOI: $10.1063 / 1.2829388$

[12] Chen G., Wang H., Zhao W.: Fabrication of highly ordered polymer/graphite flake composite with eminent anisotropic electrical property. Polymers for Advanced Technologies, 19, 1113-1117 (2008). DOI: $10.1002 /$ pat.1093

[13] Kimura T., Ago H., Tobita M., Ohshima S., Kyotani M., Yumura M.: Polymer composites of carbon nanotubes aligned by a magnetic field. Advanced Materials, 14, 1380-1383 (2002).

DOI: 10.1002/1521-4095(20021002)14:19<1380::AIDADMA1380>3.0.CO;2-V

[14] Yu G., Li X., Lieber C. M., Cao A.: Nanomaterialincorporated blown bubble films for large-area, aligned nanostructures. Journal of Materials Chemistry, 18, 728-734 (2008). DOI: $10.1039 / \mathrm{b} 713697 \mathrm{~h}$

[15] Yu G., Cao A., Lieber C. M.: Large-area blown bubble films of aligned nanowires and carbon nanotubes. Nature Nanotechnology, 2, 372-377 (2007).

DOI: $10.1038 /$ nnano.2007.150

[16] Smith P. A., Nordquist C. D., Jackson T. N., Mayer T. S., Martin B. R., Mbindyo J., Mallouk T. E.: Electricfield assisted assembly and alignment of metallic nanowires. Applied Physics Letters, 77, 1399-1401 (2000).

DOI: $10.1063 / 1.1290272$

[17] Long D. P., Lazorcik J. L., Shashidhar R.: Magnetically directed self-assembly of carbon nanotube devices. Advanced Materials, 16, 814-819 (2004). DOI: $\underline{10.1002 / \mathrm{adma} .200306388}$
[18] Abe M., Yamamoto A., Orita M., Ohkubo T., Sakai H., Momozawa N.: Control of particle alignment in water by an alternating electric field. Langmuir, 20, 70217026 (2004). DOI: $10.1021 / 1 \mathrm{a} 0490801$

[19] Kim G. H.: Thermo-physical responses of polymeric composites tailored by electric field. Composites Science and Technology, 65, 1728-1735 (2005).

DOI: $10.1016 /$ j.compscitech.2005.02.013

[20] Wang H., Zhang H., Zhao W., Zhang W., Chen G.: Preparation of polymer/oriented graphite nanosheet composite by electric field-inducement. Composites Science and Technology, 68, 238-243 (2008). DOI: 10.1016/j.compscitech.2007.04.012

[21] Garmestani H., Al-Haik M. S., Dahmen K., Tannenbaum R., Li D., Sablin S. S., Hussaini M. Y.: Polymermediated alignment of carbon nanotubes under high magnetic field. Advanced Materials, 15, 1918-1921 (2003).

DOI: $10.1002 /$ adma.200304932

[22] Hu Y., Wang Y. L., Gong X. L., Gong X. Q., Zhang X. Z., Jiang W. Q., Zhang P. Q., Chen Z. Y.: New magnetorheological elastomers based on polyurethane/Sirubber hybrid. Polymer Testing, 24, 324-329 (2005). DOI: $10.1016 /$ j.polymertesting.2004.11.003

[23] Zhao N., Gao M.: Magnetic janus particles prepared by a flame synthetic approach: Synthesis, characterizations and properties. Advanced Materials, 21, 184187 (2009). DOI: 10.1002/adma.200800570

[24] Wu J., Gong X., Fan Y., Xia H.: Physically crosslinked poly(vinyl alcohol) hydrogels with magnetic field controlled modulus. Soft Matter, 7, 6205-6212 (2011). DOI: $10.1039 / \mathrm{c} 1 \mathrm{sm} 05386 \mathrm{~h}$

[25] Takahashi T., Murayama T., Higuchi A., Awano H., Yonetake K.: Aligning vapor-grown carbon fibers in polydimethylsiloxane using dc electric or magnetic field. Carbon, 44, 1180-1188 (2006). DOI: 10.1016/j.carbon.2005.10.055 